\title{
Controlled Synthesis of Hyperbranched Poly (ester)s From Biorenewable Monomers for the Delivery of Therapeutic Agents
}

\author{
Tracy Zhang ${ }^{1,2}$, Patrick B Smith ${ }^{2}$ and Bob A Howell ${ }^{1 *}$ \\ ${ }^{1}$ Department of Chemistry, Science of Advanced Materials, Center for Applications in Polymer Science, Central Michigan University, USA \\ ${ }^{2}$ Science of Advanced Materials, Department of Chemistry, Michigan State University - St. Andrews, USA
}

*Corresponding author: Bob A Howell, Science of Advanced Materials, Center for Applications

Received Date: July 16, 2019

in Polymer Science, Department of Chemistry, Central Michigan University, USA.

Published Date: July 24, 2019

Abstract

A series of hyperbranched poly(ester)s (HBPEs) has been prepared from renewable bio monomers using the Macosko-Miller conditional probability model (appropriate for monomers containing functional groups of unequal reactivity) to select ratios of reactants (ratios of reactive functional groups) to permit high monomer conversion without gelation and to assure the presence of specific reactive end groups. These materials have been fully characterized using spectroscopic, chromatographic and thermal methods and may serve as excellent platforms for the attachment of various therapeutic agents. Even though the conjugates were not water soluble, release of the active agents from fine suspensions of the conjugates occurred readily by hydrolysis in aqueous media under enzyme-catalyzed hydrolysis. The rate of release of the therapeutic agent from the suspension was found to be zero order (linear with time) and can be tuned by altering the composition of the HBPE.

\section{Introduction}

The development of polymers [1-13] and polymer additives [14-27] based on renewable biosources is of increasing interest and continues to grow at an impressive rate ( $20 \% /$ year) [28]. These developments are driven by heightened concern for sustainability, environmental quality, and human health. Starting materials derived from natural sources are generally readily available at modest cost, are usually nontoxic (sometimes are food constituents), are renewable annually, and are often biodegradable $[29,30]$. Further, the cost of these materials is independent of fluctuations in petrochemical markets.

Esterification is widely used for the conversion of biomaterials to useful polymers or polymer additives. In particular, hyperbranched poly(ester)s derived from biomonomers offer great potential as platforms for the controlled release of active agents. While they are not as structurally uniform as their counterparts, the dendrimers, they offer ease of synthesis and a high density of reactive end group functionality. These materials are often prepared from multifunctional alcohols and difunctional carboxylic acids and have long been the base for alkyd coatings. For coating applications, the avoidance of gelation is not a concern. However, for the preparation of oligomers useful for the delivery of active agents, gelation must be avoided both to provide a workable (soluble) material and to expose a large number of reactive end groups. Traditionally, conditions to avoid gelation have been determined empirically by varying reactant concentrations, reaction times, temperature or other reaction parameters [31-37]. This approach precludes reproducibility of polymer molecular weight, dispersity, structure or level of end group functionality and is not amenable to scale-up. A more rational synthetic method is required [38]. The ability to predict the structural properties of hyperbranched poly(ester)s from an initial predefined set of reactants and experimental conditions would reduce the time and cost associated with the synthesis and would greatly improve the quality and reproducibility of the materials produced. For the polymerization of monomers containing functionality of equal reactivity, e.g., pentaerythritol and succinic acid, the Flory-Stockmayer gelation theory may be used to select appropriate monomer ratios to assure a well-defined, non-gelling structure at high conversion [39-41]. For the polymerization of monomers containing functionality of different reactivity, e.g., glycerol (the primary hydroxyl groups of glycerol are approximately 1.4 times as reactive in esterification 
as is the secondary hydroxyl group) with adipic acid, the FloryStockmayer approach is inadequate. However, the Macosko-Miller conditional probability model can be used to predict the properties of hyperbranched poly(ester)s from monomers containing functionality of unequal reactivity, as a function of the extent of reaction [42-46]. The utility of these approaches for the controlled synthesis of well-defined hyperbranched poly(ester)s from several sets of biomonomers has been demonstrated $[38,46]$. The reactive end groups of these materials have been used for the attachment of various therapeutic agents. Sustained release of the active agents has been established [47].

\section{Experimental}

\section{Materials}

Common solvents and reagents were obtained from Thermo Fisher Scientific or the Aldrich Chemical Company. Glycerol, pentaerythritol, Naproxen, salicylic acid, succinic acid and adipic acid were from the Aldrich Chemical Company.

\section{Instrumentation and characterization}

All materials were thoroughly characterized using previously described spectroscopic and thermal methods [38,46]. The structure of the hyperbranched poly(ester)s was determined in detail using carbon-13 nuclear magnetic resonance spectroscopy $\left({ }^{13} \mathrm{C}\right.$ NMR) and size exclusion chromatography [46]. Nuclear magnetic resonance (NMR) spectra were obtained using a Bruker Avance $300 \mathrm{MHz}$ spectrometer. Proton and carbon chemical shifts are reported in parts-per-million $(\delta)$ with respect to tetramethyl silane (TMS) as an internal reference $(\delta=0.00)$. Quantitative ${ }^{13} \mathrm{C}$ NMR spectra were obtained using a $90^{\circ}$ pulse width, a pulse repetition time of 10 seconds, gated decoupling without NOE, a sweep width of $31 \mathrm{KHz}, 13.1 \mathrm{~K}$ points, $3.0 \mathrm{~Hz}$ line broadening. The ${ }^{1} \mathrm{H}$ NMR spectra were obtained using a $10^{\circ}$ pulse width and a 5 second repetition rate.

SEC was performed using a Malvern GPC max instrument equipped with low angle light scattering and refractive index detectors. The instrument was fitted with two Agilent PL gel $3 \mu \mathrm{m}$ MIXED-E columns in series. The eluent was THF at a flow rate of 1 $\mathrm{mL} \mathrm{min}^{-1}$. Typical concentration of the samples was $1.0 \mathrm{mg} \mathrm{mL}^{-1}$.

High performance liquid chromatography (HPLC) was utilized to monitor the release of the therapeutic agents from the hyperbranched poly(ester) conjugates [47]. A Waters Breeze 2 system (2707 autosampler, a 1525 gradient pump, a $5 \mathrm{CH}$ column oven at $35^{\circ} \mathrm{C}$, a Waters Symmetry C18, $3 \mu$ column ( 3.0 by $150 \mathrm{~mm}$ ), and a $2489 \mathrm{UV} /$ visible detector) was used to monitor the release. A solvent gradient from $90 / 10$ to $0 / 100$ water/acetonitrile was used for an elution profile. A pH of 2.0 was maintained by addition of an aqueous phosphoric acid solution.

\section{Synthesis}

Glycerol/adipic acid hyperbranched poly(ester): The Macosko-Miller model was used to select the ratio of reactants (ratio of reactive functional groups) to permit high monomer conversion without gelation and to assure the presence of hydroxyl end groups. General methods of synthesis and characterization have been described [46]. The glycerol-adipic acid HBPEs containing hydroxyl end groups were prepared by melt polymerization at $150{ }^{\circ} \mathrm{C}$ using dibutyltin oxide as catalyst. The glycerol/succinic acid HBPEs were prepared using a similar process.

Salicylic acid attachment (glycerol-AA-SA): Salicylic acid was bonded to the glycerol-adipic acid HBPE by esterification as previously described [47]. Salicylic acid was attached to the hydroxyl end capped HBPE using P-toluenesulfonic acid as catalyst in triglyme solution at $150{ }^{\circ} \mathrm{C}$. The polymer conjugate was isolated from the triglyme solution by precipitation with diethyl ether. Naproxen was also attached through esterification.

Salicylic acid and naproxen release rate: The rate of hydrolysis of the HBPE conjugate was determined by placing 8.0 $\mathrm{mg}$ of the polymer conjugate into a vial with $7.6 \mathrm{~mL}$ of $\mathrm{pH} 7.0$ aqueous buffer to create a polymer dispersion of $1 \mathrm{mg} / \mathrm{mL}$ with the subsequent addition of $0.4 \mathrm{~mL}$ of rat liver microsomes.

The vial containing the buffer and polymer conjugate was inserted into a $37^{\circ} \mathrm{C}$ water bath and stirred vigorously for exactly 15 minutes, after which the stirrer was turned down to its lowest setting and the microsomes were added. The stirrer was turned down so as not to degrade the enzymes. Samples $(1.0 \mathrm{~mL})$ were taken directly from the reaction vial as a function of time, placed in 2-mL vials containing $200 \mu \mathrm{L}$ of THF to denature the enzyme and analyzed by HPLC to determine the amount of therapeutic agent released as a function of time.

The Naproxen conjugate was a solid at room temperature so $8 \mathrm{mg}$ of this polymer conjugate was swollen $18 \mu \mathrm{L}$ of DMSO with heating to facilitate its dispersion in the buffer solution. Rat liver microsomes $(400 \mu \mathrm{L})$ were also used for this hydrolysis.

\section{Results}

The glycerol- adipic acid and glycerol-succinic acid HBPEs were characterized using ${ }^{13} \mathrm{C}$ NMR spectroscopy and SEC [46]. These techniques, together with Miller-Macosko models, were shown to thoroughly define the structure of glycerol-adipic acid HBPEs.

The primary and secondary hydroxyl units of glycerol have different reactivity toward esterification, which will be discussed later. Nevertheless, glycerol can be substituted in one of five different ways for esterification with either succinic acid (SuA) or adipic acid (AA) as shown in Table 1. The substitution patterns include the primary monoester which is a terminal unit labeled $\mathrm{TG}$, the secondary monoester, which is also a terminal unit, $\mathrm{T}_{1,3}$, the primary-primary diester linear unit, $\mathrm{L}_{1,3}$, the primary-secondary diester linear unit, $\mathrm{L}_{1,2}$ and the trisubstituted dendritic unit, D [48]. The ${ }^{13} \mathrm{C}$ NMR spectra for all these structures have been assigned [46] and were used to quantify the glycerol substitution pattern and the extent of reaction for the minor (carboxyl) species, $\mathrm{Pb}$, and the mole ratio of the monomer building blocks, both those incorporated into the HBPE and unreacted. These values were used as inputs to the Miller-Macosko conditional probability model to predict both the 
structure and molecular weight of HBPEs prepared with varying monomer stoichiometry [42-46].

The conditional probability model utilizes a series of "superspecies", $G_{00}$ through $G_{21}$, defined in Figure 1 and correspond to the glycerol substitution patterns of Table $1 . G_{00}$ represents unsubstituted glycerol, the primary and secondary monoesters, $G_{10}$ and $G_{01}$ correspond to $\mathrm{Tg}$ and $T_{1,3}$, respectively, the two diesters, primary-primary, $G_{20}\left(L_{1,3}\right)$, and primary-secondary, $G_{11}\left(L_{1,2}\right)$ and the triester, $G_{21}$ (D). The distribution of glycerol super-species was quantitatively determined using ${ }^{13} \mathrm{C}$ NMR. The weight-average molecular weight and dispersity of these species were predicted using conditional probability to randomly combine the glycerol super-species with adipic acid. The model is able to accommodate the difference in primary and secondary hydroxyl reactivity as well as substituent effects for the glycerol hydroxyl reactivity upon substitution of one of the other three hydroxyl groups because the distribution of glycerol ester species was quantitatively determined. The model assumes that the adipic acid carboxyl groups possess equal reactivity, react independently and that there were no intramolecular reactions.

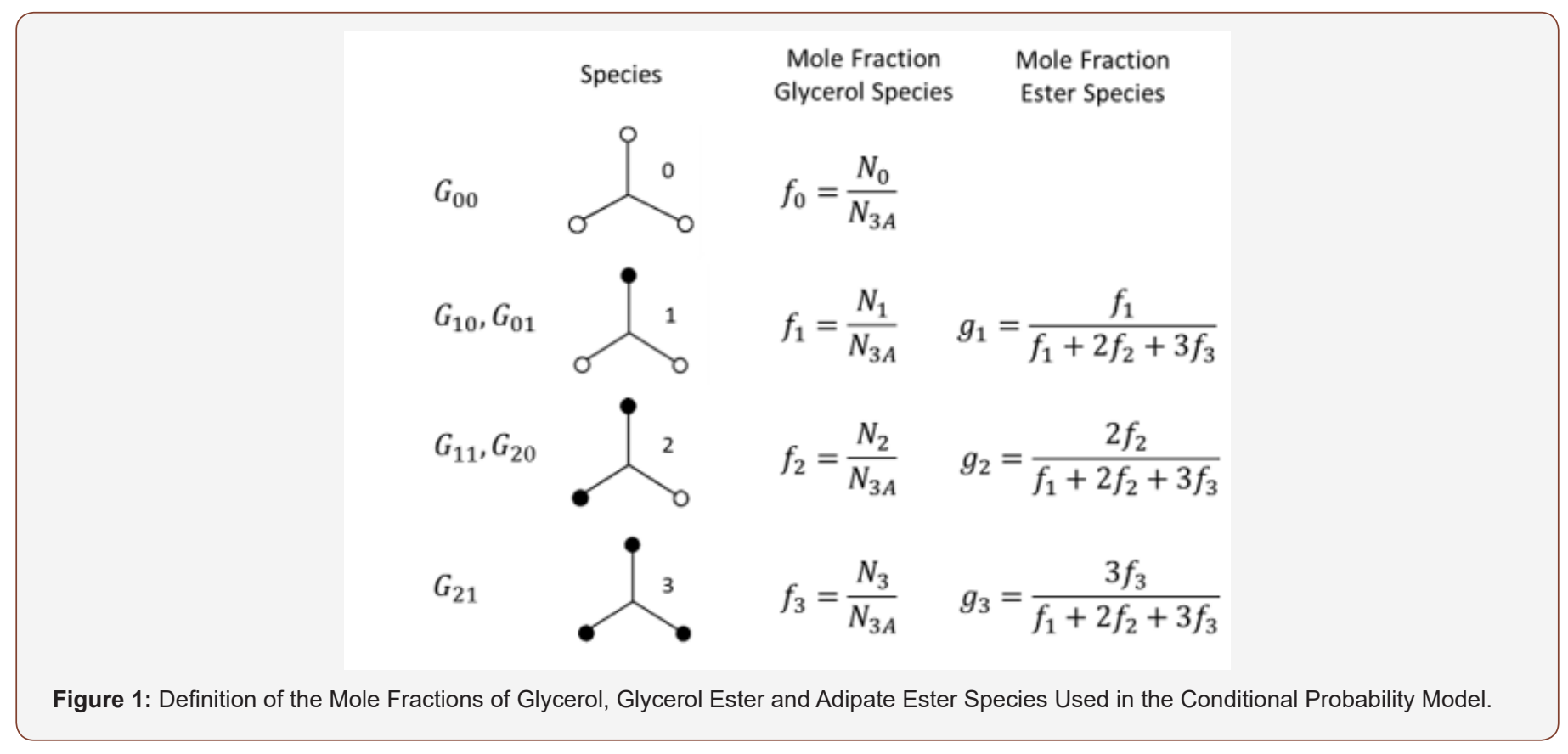

Table 1: Substituent Structures and Nomenclature for Glycerol-Adipic Acid HBPE.<smiles>OCC(O)CO</smiles><smiles>[R]OCC(O)CO</smiles><smiles>[R]OCC(O)CO[R]</smiles><smiles>[R]OCC(CO)O[R]</smiles><smiles>[R]OCC(CO[R])O[R]</smiles> 
The mole fraction of glycerol, $\mathrm{f}_{0}$, is equal to the moles of glycerol divided by the total number of moles of all glycerol species, $\mathrm{N}_{3 \mathrm{~A}^{\prime}}$ as shown in Figure 1. The mole fraction of the monoesters, diesters and trimesters are defined as $\mathrm{f}_{1}, \mathrm{f}_{2}$ and $\mathrm{f}_{3}$, respectively. The mole fraction of the ester species is then given by $g_{1}$ though $g_{3}$. The molecular weight values for each glycerol species are given in the final column of Figure 1. A molecule of water is lost for each esterification reaction, so as a housekeeping tool, the molecular weight of the glycerol unit is assumed in the calculation to lose 1 mass unit and the acid group loses 17 mass units.

The value of the Miller-Macosko conditional probability model is that it is possible to accurately predict the molecular weight and dispersity of the HBPEs using only the initial monomer stoichiometry for polymerizations taken to high extent of reaction. Accurate molecular weight prediction is not possible with the more conventional Flory-Stockmayer (F-S) model, which is only valid for monomers with functional groups of equal reactivity [3941]. The Miller-Macosko conditional probability model makes it possible to target a given molecular weight and structure for HB polymers simply by selecting the initial monomer stoichiometry for polymerizations taken to high conversion. It gives control and reproducibility to the synthesis of these materials.

In order to demonstrate the power of the conditional probability model to target molecular weight in this fashion, a series of HBPEs of varying monomer stoichiometry, or more precisely, functional group stoichiometry, [-OH] / [-COOH], varying from 1.52 to 2.16 were produced. ${ }^{13} \mathrm{C}$ NMR spectroscopy was used to quantify the ratio of the substituted glycerol species and the degree of branching (DB\%) as a function of stoichiometry for these HBPEs (Table 2).

Table 2: The Initial Functional Group Stoichiometries $[-\mathrm{OH}] /[-\mathrm{COOH}]$ for the Series of HBPEs, Mole Ratio of Substituents and Degree of Branching.

\begin{tabular}{|c|c|c|c|c|c|c|c|}
\hline Stoichiometry & Gly. & $\mathbf{T}_{G}$ & $\mathbf{T}_{13}$ & $\mathbf{L}_{13}$ & $\mathbf{L}_{12}$ & $\mathbf{D}$ & $\mathbf{D B}_{\mathbf{1}}$ \\
\hline 2.16 & 0.15 & 0.37 & 0.05 & 0.24 & 0.13 & 0.06 & 24 \\
\hline 2 & 0.12 & 0.37 & 0.05 & 0.26 & 0.14 & 0.06 & 23 \\
\hline 1.93 & 0.14 & 0.34 & 0.05 & 0.26 & 0.12 & 0.09 & 32 \\
\hline 1.74 & 0.08 & 0.31 & 0.05 & 0.31 & 0.15 & 0.1 & 30 \\
\hline 1.69 & 0.08 & 0.3 & 0.04 & 0.32 & 0.15 & 0.11 & 32 \\
\hline 1.52 & 0.04 & 0.26 & 0.03 & 0.34 & 0.18 & 0.15 & 36 \\
\hline
\end{tabular}

The degree of branching was determined from the following equation [49]:

$$
D B \%=\frac{2 D}{2 D+\left(L_{1,2}+L_{1,3}\right)} \times 100
$$

The values for $\mathrm{D}, \mathrm{L}_{1,2}$ and $\mathrm{L}_{1,3}$ as a function of stoichiometry were obtained directly from the areas of the ${ }^{13} \mathrm{C}$ NMR spectra and are given in Table 2.

The extent of reaction of the carboxyl functionality $(\mathrm{Pb})$ quantified using ${ }^{13} \mathrm{C}$ NMR spectroscopy, the weight-average molecular weight $(\mathrm{Mw})$ determined using SEC-LALS, the weightaverage molecular weight predicted using the Miller-Macosko model using the ${ }^{13} \mathrm{C}$ NMR-determined super-species distribution, are summarized in Table 3. The agreement between predicted molecular weight values (Miller-Macosko) and those determined by SEC-MALLS is excellent. The fact that the F-S model fails to correctly predict the MW values and gel points for this series of HBPEs is demonstrated by the last column of Table 3. The rate of esterification for the secondary hydroxyl is about one third that

Table 3: Determined (SEC-LALS) and Predicted Mw (Miller-Macosko Model) for HBPEs with Varying Reactant Stoichiometry, and the Extent of Reaction Gel-point Predicted by the Flory-Stockmayer Model.

\begin{tabular}{|c|c|c|c|c|}
\hline Stoichiometry & Determined Mw (SEC-LALS) & Predicted Mw (Miller-Macosko) & Pb (NMR) & Pb, gel (Flory-Stockmayer) \\
\hline 2.16 & 1100 & 1569 & 0.96 & no gel \\
\hline 2 & 1250 & 1454 & 0.93 & 1 \\
\hline 1.93 & 2100 & 2015 & 0.91 & 0.98 \\
\hline 1.74 & 2400 & 2938 & 0.92 & 0.93 \\
\hline 1.69 & 4900 & 3545 & 0.91 & 0.92 \\
\hline 1.52 & 14000 & 14492 & 0.9 & 0.54 \\
\hline
\end{tabular}




\section{Controlled release from the HBPE conjugate}

Salicylic acid as an active agent: Salicylic acid is a common therapeutic used for the treatment of acne and inflammation [5052]. It is often administered at relatively high doses directly onto the skin to treat acne and often gives rise to skin irritation due its acidic nature. The ability to time-release salicylic acid would enhance the duration of its effectiveness while minimizing skin irritation.

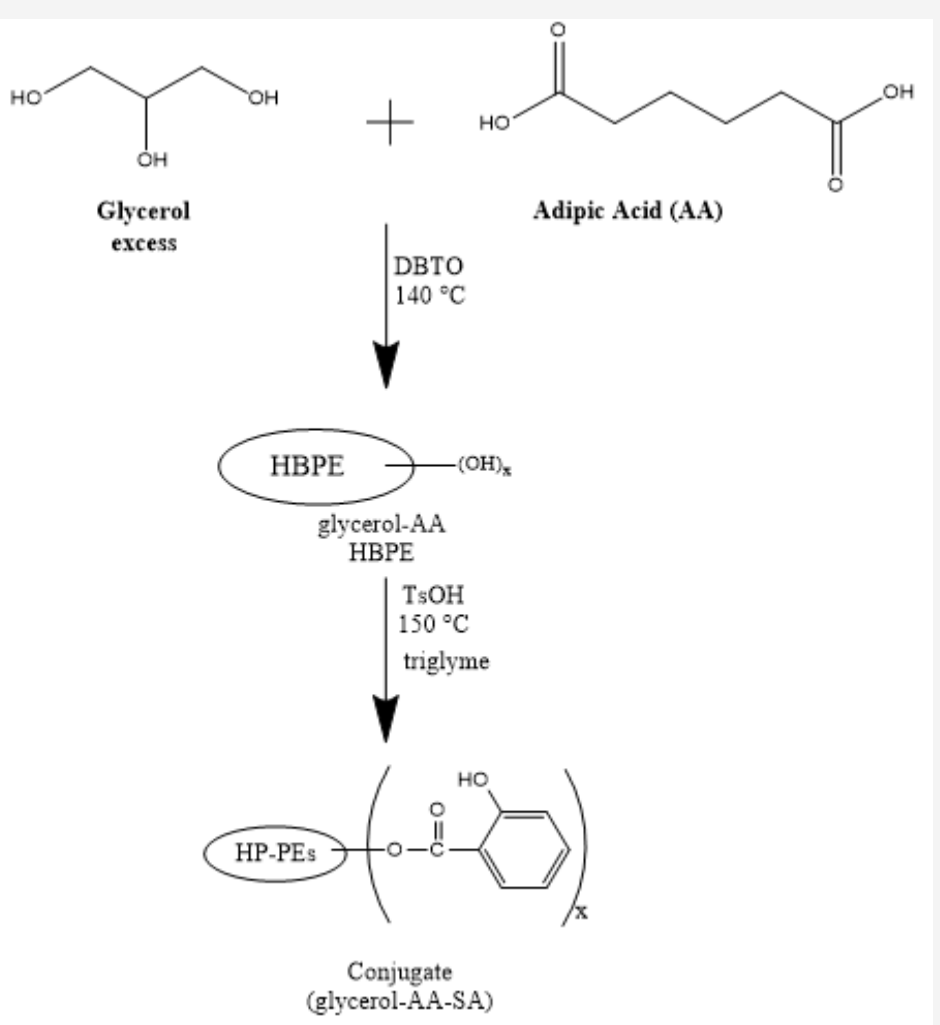

Scheme 4: Synthesis of the Salicylic Acid Conjugate of a Glycerol-Adipic Acid HBPE.

A time-release formulation was developed by covalently bonding salicylic acid to the glycerol-adipic acid HBPE with hydroxyl end groups by esterification through its carboxylic acid functionality as shown in Scheme 4. The resulting polymer conjugate possessed a molecular weight of approximately 3,200 g/mol (SEC-MALS).

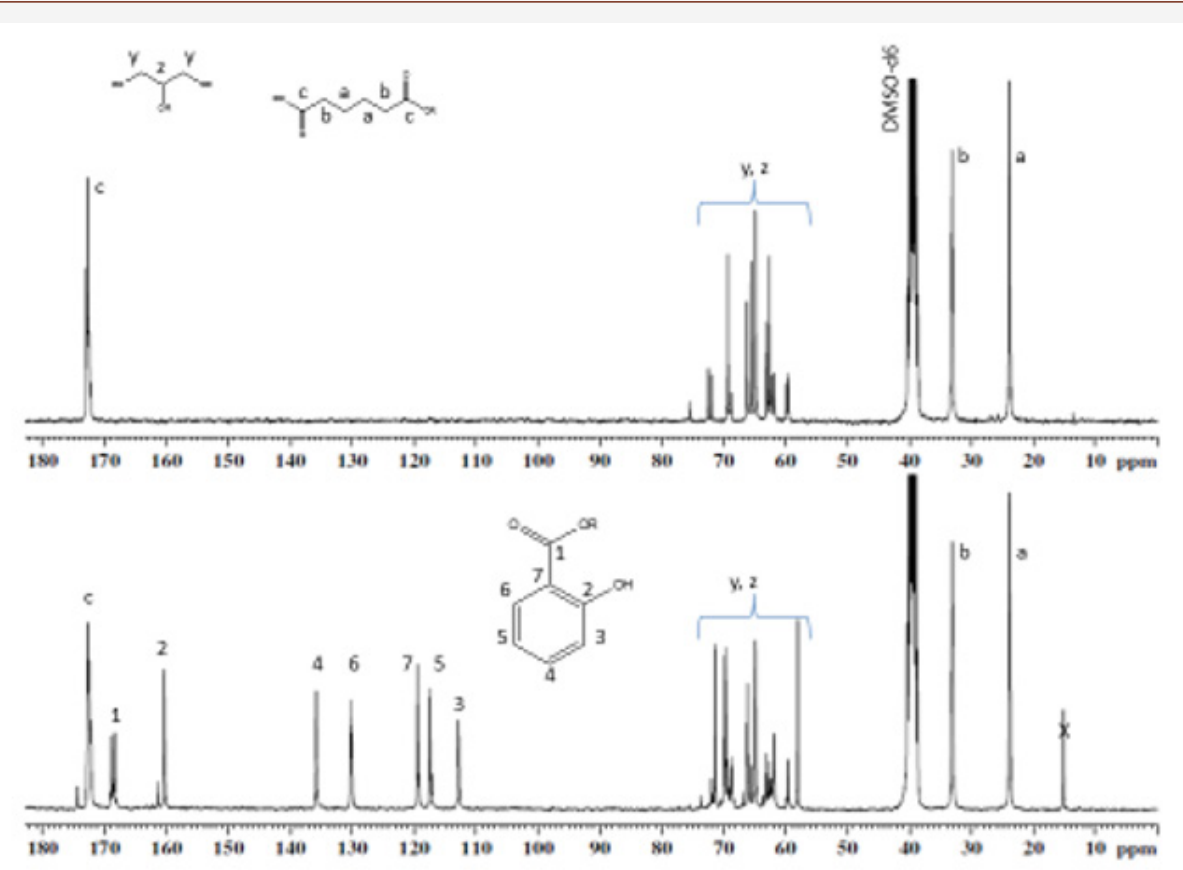

Figure 7: Quantitative 13C NMR Spectrum of a Glycerol-Adipic Acid HBPE Containing Hydroxyl Endgroups (top) and its Salicylic Acid Conjugate (bottom). 
The quantitative ${ }^{13} \mathrm{C}$ NMR spectrum of the original HBPE, shown in Figure 7 (top spectrum), contains resonances for the ester carbonyl at $\delta 172.6$ and the methylenes, "d" and "e" at about 33 and $24 \mathrm{ppm}$. The spectrum of the salicylic acid conjugate (Figure 7 bottom) contains a resonance for the ester carbonyl of the polymer at $\delta 172.7$, one for the ester carbonyl from the conjugate at $\delta 168.3$, as well as the aromatic carbon atoms of salicylic acid which are labeled on the spectrum. A small acid carbonyl of adipic acid just downfield from carbon "c" was due to a slight amount of hydrolysis of the HBPE during the esterification of the salicylic acid. The small peak just downfield of carbon "2" of salicylic acid was due to the presence of a small amount of free salicylic acid.
The glycerol-AA-salicylic acid HBPE conjugate was not water soluble. Rather, it was suspended in water by vigorously stirring the mixture. Care was taken to stir each mixture at a constant rate and time to achieve reproducible suspensions and thereby, release kinetics. The enzymatic hydrolysis of the conjugate was performed in duplicate in order to evaluate the reproducibility of the experiment. Figure 8 shows that the hydrolysis rates were quite reproducible. Additionally, the salicylic acid release rates for both conjugates were nearly linear within the two-hour testing window, indicating a fairly constant release rate that is consistent with zero order release kinetics.

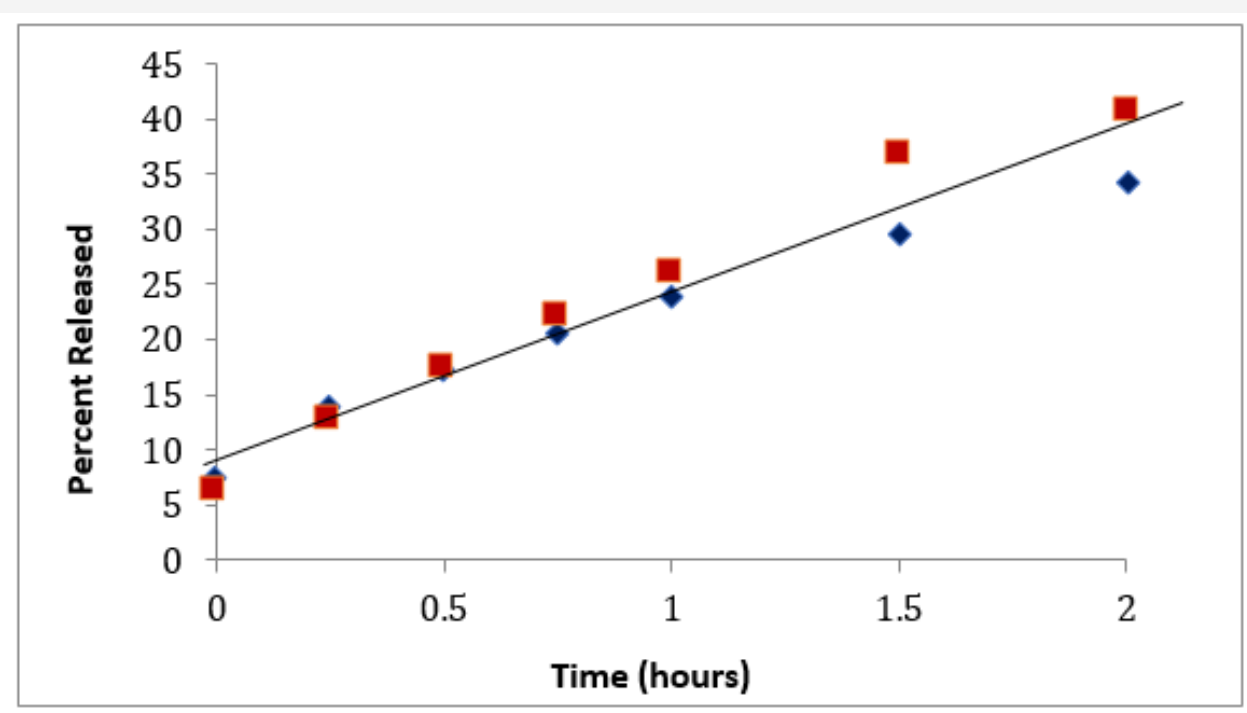

Figure 8: Salicylic Acid Release Rates from the Glycerol-AA-Salicylic Acid Conjugate from Duplicate Hydrolysis Experiments.

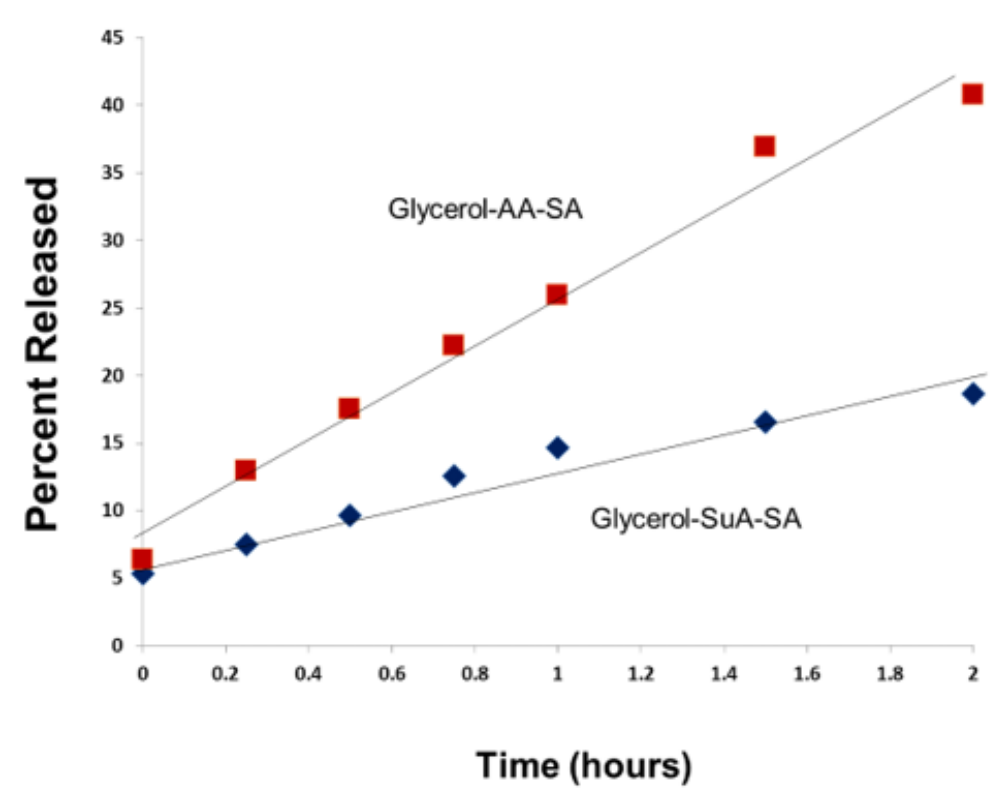

Figure 9: Salicylic Acid Release Rate from Glycerol-AA-SA and Glycerol-SuA-SA HBPE Conjugates.

An HBPE conjugate of glycerol-succinic acid-salicylic acid was shown to release salicylic acid much more slowly than the glyceroladipic acid-salicylic acid conjugate (Figure 9). The salicylic acid release from the glycerol-succinic acid-salicylic acid conjugate was also nearly linear with time. 
A control study of the rate of release of salicylic acid from the glycerol-adipic acid HBPE conjugate, in the presence and absence of rat liver microsomes (Figure 10) was conducted. These data clearly show that the rate of salicylic acid release from the conjugate is orders of magnitude slower without the catalytic effect of rat liver microsomes.

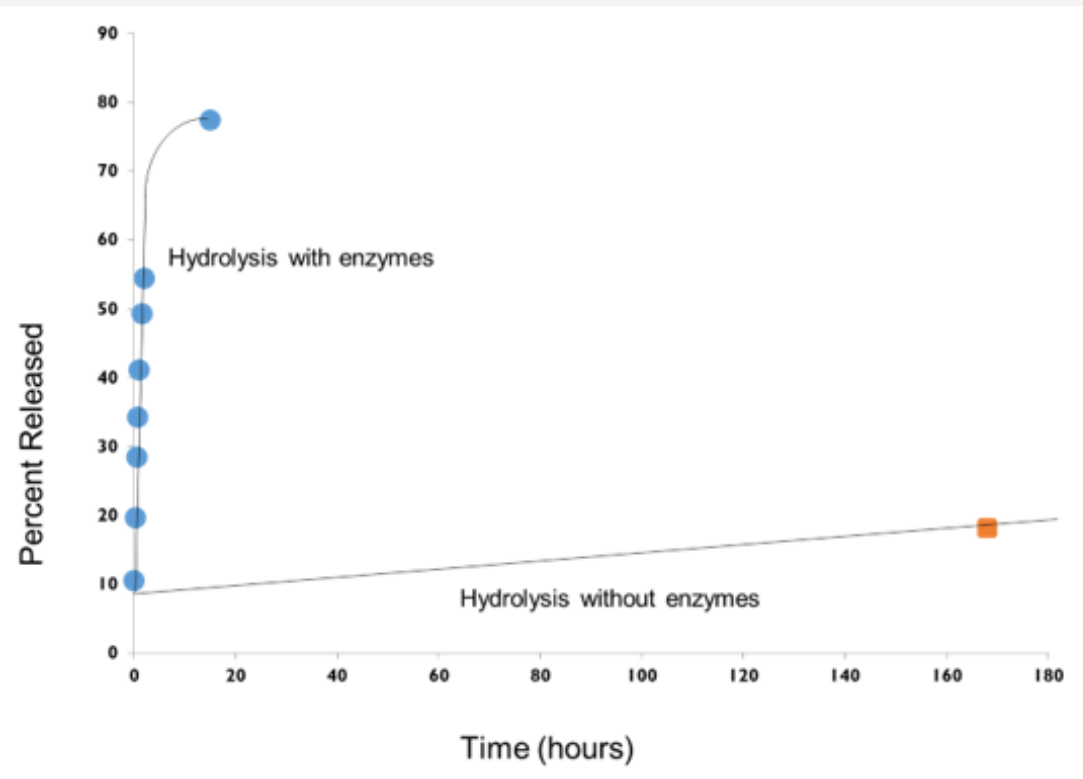

Figure 10: The Release of Salicylic Acid from the Glycerol-Adipic Acid-Salicylic Acid Conjugate in the Presence and Absence of Rat Liver Microsomes.

The release rate of salicylic acid from a glycerol-adipic acid HBPE and that of glycerol-succinic acid was shown to be quite different indicating the ability to control the rate of release based on HBPE composition. The release rates from both HBPEs were also linear with time suggesting zero order kinetics for the release.

The zero-order kinetics were probably due to the fact that the conjugates were not water soluble but were rather suspensions in the degradation solution. The suspended conjugate particles were probably eroded from the outer surface in the presence of enzymes, giving rise to process known to possess zero order kinetics for the release of an active [52].

The cause for the release of salicylic acid from the glycerolAA HBPE conjugate being faster than that from the glycerol-SuA HBPE conjugate was probably due to the relative particle size of the two dispersions. The glycerol-SuA HBPE conjugate was a stiffer material and more difficult to disperse and, therefore, probably had a larger particle size.

\section{Naproxen as an active agent}

Naproxen is a nonsteroidal anti-inflammatory drug, used for the treatment of swelling, stiffness and pain. A conjugate was prepared by bonding Naproxen (its structure is shown in Figure 11) by esterification to a hydroxyl terminated HBPE of glycerolAA. This glycerol-adipic acid- Naproxen conjugate also releases the active agent by enzymatic hydrolysis in the presence of rat liver microsomes. Duplicate kinetic runs for the glycerol-AA-Naproxen conjugate are shown in Figure 11. The release of Naproxen was significantly slower than that for the salicylic acid conjugates, but linear release rates were again observed, suggesting the same mechanism for release.

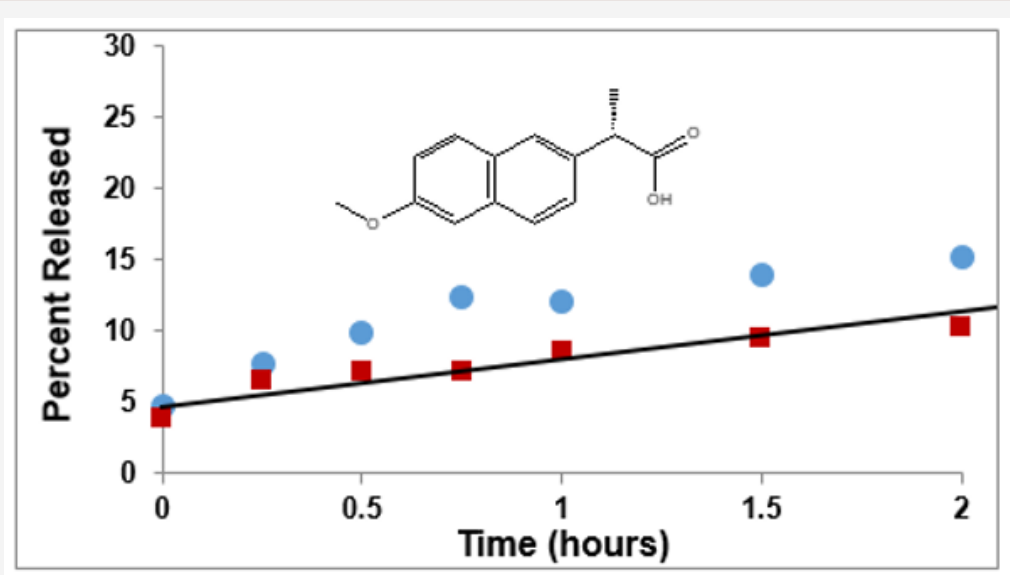

Figure 11: Release of Naproxen from Capped Glycerol-AA HBPE, Duplicate Experiments. 


\section{Conclusion}

A series of hyperbranched polyesters from glycerol and adipic acid were prepared by polyesterification as a function of initial monomer stoichiometry, providing an excellent sample set to demonstrate the power of concerted characterization and modeling tools. The ${ }^{13} \mathrm{C}$ NMR spectra were completely assigned in terms of glycerol and the five glycerol ester species. The ratio of these species was quantitatively determined as a function of initial monomer stoichiometry for the set of HBPEs and the MW was determined for each. The weight-average molecular weights were predicted from the measured distribution of glycerol ester species using the Miller-Macosko conditional probability model for this set of HBPEs of different stoichiometry. The model accounted for the unequal reactivity of the glycerol primary and secondary alcohol groups, as well as possible substituent effects. The MillerMacosko conditional probability model was shown to accurately predict the measured molecular weights. This model facilitated the preparation of HBPEs of well-defined and reproducible structure simply from the initial monomer stoichiometry.

Hyperbranched polyesters from glycerol-adipic acid and glycerol-succinic acid were then converted to conjugates with two therapeutic agents; salicylic acid and Naproxen. These HBPEs were shown to provide an effective biobased platform for the controlled release of bioactive agents. This delivery platform has the advantage that it is nontoxic, inexpensive and versatile, allowing for tunable time-delivery formulations. The therapeutic agents, salicylic acid and Naproxen, were covalently bonded to the HBPE by esterification and then released through enzymatic hydrolysis using rat liver microsomes. The loading level and rate of release were controlled by proper choice of HBPE structure and molecular weight and catalyst level.

\section{Acknowledgement}

The authors would like to thank Dr. Thomas Chamberlain and Dendritech, Inc. for assistance with HPLC analysis of the release rates of the salicylic acid conjugates and Michigan State University and Central Michigan University for funding.

\section{Conflict of Interest}

No conflict of interest.

\section{References}

1. A Gandini (2008) Polymers from Renewable Resources: A Challenge for the Future of Macromolecular Materials. Macromolecules 41(24): 94919504.

2. GQ Chen, MK Patel (2012) Plastics Derived from Biological Sources: Present and Future: A Technical and Environmental Review. Chem. Rev 112(4): 2082-2099.

3. J Kuczynski, DJ Boday (2012) Biobased Materials for High-end Electronics Applications. Int J Sust Dev World 19: 557-563.

4. MR Reddy, S Vivekanandhan, M Misra, SK Bhatia, AK Mohanty (2013) Biobased Plastics and Bionanocomposites: Current Status and Future Opportunities. Prog Polym Sci 38(10-11): 1653-1689.

5. R Mulhaupt (2013) Green Polymer Chemistry and Bio-Based Plastics: Dreams and Reality. Macromol Chem Phys 214(2): 159-174.
6. PFH Harmsen, MM Hackman, HL Bos (2014) Green Building Blocks for Bio-based Plastics. Biofuels Bioprod Bioref 8(3): 306-324.

7. Y Jiang, GORA van Ekenstein, AJJ Woortman, K Loos (2014) Fully Biobased Unsaturated Aliphatic Polyesters from Renewable Resources: Enzymatic Synthesis, Characterization, and Properties. Macromol Chem Phys 215(22): 2185-2197.

8. T Iwata (2015) Biodegradableand Bio-Based Polymers: Future Prospects of Eco-Friendly Plastics. Angew Chem Int Ed 54(11): 3210-3215.

9. A Pellis, EH Acero, L Gardossi, V Ferrario, GM Guebitz (2016) Renewable Building Blocks for Sustainable Poly(ester)s:New Biotechnological Routes for Greener Plastics. Polym Int 65(8): 861-871.

10. M Hillmyer (2017) The Promise of Plastics from Plants. Science 358(6365): 868-870.

11. TJ Farmer, JW Comerford, A Pellis, T Robert (2018) Post-polymerization Modification of Bio-based Polymers: Maximizing the High Functionality of Polymers Derived from Biomass. Polym Int 67(7): 775-789.

12. H Xie, L Wu, BG Li, P Du Bois (2019) Modification of Poly (ethylene, 2,5-furandicarboxylate) with Biobased 1,5-Pentanediol: Significantly Toughened Copolymers Retaining Strength and Oxygen Barrier Properties. Biomacromolecules 20(1): 318-325.

13. $\mathrm{T}$ Goto, $\mathrm{T}$ Iwata, $\mathrm{H}$ Abe (2019) Synthesis and Characterization of Biobased Polyesters Containing Anthraquinones Derived from Gallic Acid", Biomacromolecules 20(1): 318-325.

14. M Malinconico, P Cerruti, G Santagata, B Immirizi (2014) Natural Polymers and Additives in Commodity and Specialty Applications: A Challenge for the Chemistry of the Future. Macromol Symp 337(1): 124133.

15. J Chen, X Nie, J Jiang (2018) Synthesis and Application of a Novel Environmental Cardanol-based Plasticizer as a Secondary or Main Plasticizer for Poly (vinyl chloride). Polym Int 67(3): 269-275.

16. K Zhang, XP Chang, F Cheng, Y Lin, M Zhou, et al. (2018) Poly(citrate glyceride): a Hyperbranched Polyester for Starch Plasticization. Polym Int 67(4): 399-404.

17. BA Howell, W Sun (2018) Biobased Plasticizers from Tartaric Acid, an Abundantly Available, Renewable Material. Ind Eng Chem Res 57: 15234-15242.

18. N Gama, R Santos, B Godinho, R Silva, A Ferreira (2019) Triacetin as a Secondary PVC Plasticizer. J Polym Environ 27: 1294-1301.

19. BA Howell, ST Lazar (2018) Biobased Plasticizers from Carbohydratederived 2, 5-bis(Hydroxymethyl)furan. Ind Eng Chem Res 58(3): 12221228.

20. R Sonnier, A Taquet, L Ferry, JN LopezCuesta (2018) Towards Biobased Flame Retardant Polymers. Springer International Publishing AG, Chem, Switzerland.

21. L Costes, F Laoutid, S Brohez, P Dubois (2017) Bio-based Flame Retardants: When Nature Meets Fire Protection. Mater Sci Eng 117: $1-25$.

22. YG Daniel, BA Howell (2017) Flame Retardant Properties of Isosorbide bis-Phosphorus Esters. Polym Degrad Stab 140: 25-31.

23. YG Daniel, BA Howell (2018) Phosphorus Flame Retardants from Isosorbide bis-Acrylate. Polym Degrad Stab 156: 14-21.

24. BA Howell, W Sun (2018) Biobased Flame Retardants from Tartaric Acid and Derivatives. Polym Degrad Stab 157: 199-211.

25. BA Howell, KL Oberdorfer, EA Ostrander (2018) Phosphorus Flame Retardants for Polymeric Materials from Gallic Acid and Other Naturally Occurring Multihydroxybenzoic Acids. Int J Polym Sci, pp. 12.

26. BA Howell, A Alrubayyi (2019) 2-Dopyl-1,4-di(2-dopylpropanoyl) benzene, an Effective Phosphorus Flame Retardant. Polym Degrad Stab 162: $196-200$.

27. C Hu, S Bourbigot, T Delaunay, M Collinet, S Marcillec, G Fontainea (2019) Synthesis of Isosorbide Based Flame Retardants: Application for Polybutylene Succinate. Polym Degrad Stab 164: 9-17. 
28. AL Holmberg, KH Reno, RP Wool, TH Epps III (2014) Biobased Building Blocks for the Rational Design of Renewable Block Polymers. Soft Matter 10: 7405-7424.

29. S Lambert, M Wagner (2017) Environmental Performance of Bio-Based and Biodegradable Plastics: The Road Ahead. Chem Soc Rev 46(22): 6855-871.

30. TP Haider, C Volker, J Kramm, K Landfester, FR Wurm (2019) Plastics of the Future? The Impact of Biodegradable Polymers on the Environment and on Society. Angew Chem Int Ed 58(1): 50-62.

31. JF Stumbe, B Bruchmann (2004) Hyperbranched Polyesters Based on Adipic Acid and Glycerol. Macromol Rapid Commun 25(9): 921-924.

32. VT Wyatt (2012) Lewis Acid-catalyzed Synthesis of Hyperbranched Polymers Based on Glycerol and Diacids in Toluene. J Am Oil Chem Soc 89(2): 313-319.

33. HAM Saeed, YA Eltahir, Y Xia, Y Wang (2014) Synthesis and Characterization of A2+ B4 Hyperbranched Polyesters with Hydroxyl End Groups. Adv Mater Res 937: 80-85.

34. Y Li, WD Cook, C Moorhoff, WC Huang, QZ Chen (2013) Synthesis, Characterization and Properties of Biocompatible Poly(glycerol sebacate) Pre-Polymer And Gel. Polym Int 62(4): 534-547.

35. HR Kricheldorf, G Behnken (2008) Biodegradable Hyperbranched Aliphatic Polyesters Derived from Pentaerythritol. Macromolecules 41: 5651-5657.

36. H Cao, Y Zheng, J Zhou, W Wang, A Pandit (2011) A Novel Hyperbranched Polyester Made from Aconitic Acid (B3) and Di (ethylene glycol) (A2). Polym Int 60(4): 630-634.

37. Z Fan, A Lederer, B Voit (2009) Synthesis and Characterization of A2+ B3-Type Hyperbranched Aromatic Polyesters with Phenolic End Groups. Polymer 50: 3431-3439.

38. T Zhang, BA Howell, PB Smith (2017) Rational Synthesis of Hyperbranched Poly(ester)s. Ind Eng Chem Res 56: 1661-1670.

39. PJ Flory (1952) Molecular Size Distribution in Three Dimensional Polymers. VI. Branched Polymers Containing A-R-Bf-1 Type Units. J Amer Chem Soc 74(11): 2718-2723.

40. WH Stockmayer (1943) Theory of Molecular Size Distribution and Gel Formation in Brached-chain Polymers. J Chem Phys 11(2): 45.
41. WH Stockmayer (1944) Theory of Molecular Size Distribution and Gel Formation in Branched Polymers II. General Cross Linking. J Chem Phys 12: 125 .

42. DR Miller, CW Macosko (1980) Substitution Effects in Property Relations for Stepwise Polyfunctional Polymerization. Macromolecules 13(5): 1063-1069.

43. DR Miller, CW Macosko (1978) Average Property Relations for Nonlinear Polymerization with Unequal Reactivity. Macromolecules 11(4): 656662.

44. DR Miller, CW Macosko (1976) A New Derivation of Post Gel Properties of Network Polymers. Macromolecules 9(2): 206-211.

45. CW Macosko, DR Miller (1976) A New Derivation of Average Molecular Weights of Nonlinear Polymers. Macromolecules 9(2): 199-206.

46. T Zhang, BA Howell, A Dumitrascu, SJ Martin, PB Smith (2014) Synthesis and Characterization of Glycerol-Adipic Acid Hyperbranched Polyesters. Polymer 55(20): 5065-5072.

47. T Zhang, BA Howell, SJ Martin, B Zhu, D Zhang, et al. (2018) Rational Synthesis of Biobased Hyperbranched Poly (ester) s for Sustained Delivery. in HN Cheng, RA Gross, PB Smith, Eds., Green Polymer Chemistry: New Products, Processes and Applications (ACS Symposium Series 1310) American Chemical Society, Washington DC, 13: 177-199.

48. AS Kulshrestha, W Gao, RA Gross (2005) Glycerol Copolyesters: Control of Branching and Molecular Weight Using a Lipase Catalyst. Macromolecules 38(8): 3193-3204.

49. VT Wyatt, GD Strahan (2012) Degree of Branching in Hyperbranched Poly(glycerol-co-diacid)s Synthesized in Toluene. Polymers 4(1): 396407.

50. BE Amborabé, P Fleurat Lessard, JF Chollet, G Roblin (2002) Antifungal Effects of Salicylic Acid and Other Benzoic Acid Derivatives Towards Eutypa lata: Structure-Activity Relationship. Plant Physiol Biochem 40(12): 1051-1060.

51. S Titus, J Hodge (2012) Diagnosis and Treatment of Acne. Am Fam Physician 86(8): 734-740.

52. JF Faig, KSmith, AMoretti, WYu, KEUhrich (2016) One-PotPolymerization Syntheses: Incorporating Bioactives into Poly(anhydride-esters). Macromol Chem Phys 217(16): 1842-1850. 